\title{
Value of routine histopathological examination of appendices in Hong Kong
}

\author{
W CHAN, K H FU \\ From the Department of Pathology, University of Hong Kong, Queen Mary Hospital, Hong Kong
}

SUMMARY A retrospective study of the histopathological findings of more than 11443 appendices submitted as surgical specimens over 14 years was performed in this department. In most cases routine histopathological examination added little clinically important information to other clinical and operative gross findings, but a variety of interesting and uncommon lesions were identified. In 85 cases clinically important pathological findings were first discovered on routine histopathological examination. These included enterobiasis, schistosomiasis, mucocele, trichuriasis, tuberculosis, ascariasis, endometriosis, mucinous cystadenoma, granuloma, carcinoid tumour, neuroma, clonorchiasis, primary adenocarcinoma and secondary carcinoma.

The human appendix is a supposedly functionless organ, but can cause morbidity and mortality because it is likely to become infected. ${ }^{1}$ For that reason many appendices, whether normal or abnormal, are surgically resected. In some centres the resected appendix is always submitted for histopathological examination, comprising a substantial portion of the work load of surgical pathology laboratories; in others, the appendix is sent for examination only when the operative findings are inconclusive. ${ }^{2}$ Some surgeons invert rather than resect the appendix on finding an apparently normal appendix at operation. In view of the variation in clinical practice this study was carried out to assess the value of routine histopathological examination of surgically resected appendices in Hong Kong.

This hospital is the teaching hospital of the University of Hong Kong and the only major government general hospital on Hong Kong Island that serves a population of about one million.

Hong Kong has a population of five million, of which over $98 \%$ are Chinese. ${ }^{3}$ The traditional Chinese diet is very different from the Western diet. There is some evidence to suggest that acute appendicitis is more common in Western societies because of the low residue diet. ${ }^{4}$

\section{Material and methods}

An SNOP biopsy index file was reviewed for all appendices examined during the 14 years from 1972 to

Accepted for publication 12 November 1986
$1985 .^{5}$ The diagnoses were grouped for analysis. Group 1 (normal and minor changes) included those diagnosed as normal, faecaliths, haemorrhage, congestion, lymphoid hyperplasia, involution, atrophy, obstruction, fibrous obliteration, fibrosis, fatty infiltration, diverticulum, no tumour, autolysis, and oedema. Group 2 (appendicitis and related conditions) included the following diagnoses: acute appendicitis, abscess, inflammation, active chronic inflammation, chronic inflammation, chronic abscess, rupture or perforation, gangrene, stricture, ulceration, necrosis, ischaemia and torsion.

The request forms, the pathologists' reports, and the microscopic slides from all cases with a diagnosis other than those in the above two groups were reviewed in detail. Basing on these findings, the relevance of the histopathological examination of the appendix to postoperative clinical management in individual cases was determined. Any localised lesion of the appendix that was microscopic, cured by appendicectomy, and without important clinical associations was regarded as not having benefited from routine histopathological examination. Diagnoses which were considered to be minor changes or related to acute appendicitis as listed under groups 1 and 2 were similarly regarded. Routine pathology was also taken to be of minimum value for appendices showing unusual gross features pertinent to diagnoses other than those in groups 1 and 2, when these features had been previously identified by the resecting surgeons or gynaecologists.

In some cases the diagnoses made on the appendix were also obtained by the examination of other speci- 
mens concurrently submitted; in others, the diagnosis had been made on previously submitted surgical specimens. Examination of appendices in these cases was classified as non-contributory to the final diagnoses.

The mean and median numbers of tissue blocks taken for the routine study of resected appendices were estimated by counting the number of blocks taken in 300 consecutive cases of appendicectomies in the year 1980 .

\section{Results}

A total of 12513 entries of appendicular lesions were indexed in the biopsy file between 1972 to 1985 . The original indexing diagnoses amounted to 51 . The total number of appendices examined was more than 11443 which was the sum of 1599 normal appendices and 9844 cases of acute appendicitis.

\section{GROUP 1 DIAGNOSES}

Fifteen diagnoses and 2109 entries were included in this group: normal (1599), faecaliths (203), haemorrhage (11), congestion (12), lymphoid hyperplasia (113), involution (2), atrophy (19), obstruction (2), fibruous obliteration (38), fibrosis (78), fatty infiltration (1), diverticulum (6), no tumour (22), autolysis (2), and oedema (1).

\section{GROUP 2 DIAGNOSES (APPENDICITIS AND \\ RELATED CONDITIONS)}

These included the following 13 diagnoses: acute appendicitis (9844), abscess (98), inflammation (82), active chronic inflammation (2), chronic inflammation (24), chronic abscess (3), rupture or perforation (158), gangrene (7), stricture (1), ulceration (1), necrosis (2), ischaemia (1) and torsion (1). The number of entries in group 2 amounted to 10224 .

\section{REMAINING DIAGNOSES}

The remaining 23 indexing diagnoses comprised 180 entries (table). Granulomatous lesions accounted for 27 cases. Of the 58 various parasitic infections diagnosed by examination of the appendices, there were 26 cases of enterobiasis, 20 cases of schistosomiasis, six cases of trichuriasis, five cases of ascariasis and one case of clonorchiasis.

Fifty cases of benign tumours and tumour like lesions were diagnosed: there were 26 mucoceles, one hyperplastic polyp, three adenomas, six mucinous cystadenoma, 11 carcinoid tumours, two leiomyomas and one neuroma. There were seven cases of primary adenocarcinoma of the appendix. Secondary spread to the appendix in malignant conditions included 25 cases of carcinomatous spread, two cases of malignant lymphoma, and one case of lymphoblastic leukaemic infiltration. Endometriosis was found in nine cases, and one appendix was indexed under the diagnosis of Hirschprung disease.

\section{GRANULOMATOUS INFLAMMATION}

Of the 27 appendices with granulomatous inflammation, two patients had undergone interval appendicectomy for appendicular abscess and the granulomatous inflammation was related to suture materials. In an 11 year old boy the granuloma was suspected to have formed around a pinworm. In a 16 year old girl the reporting pathologist raised the possibility of granuloma forming against a nematode. Eight cases of granulomas were interpreted to be a reaction to foreign bodies, the exact nature of which was not known. Food particles were implicated in two cases. Barium sulphate crystals were identified in one case. In the two cases of xanthogranuloma focal collections of foamy macrophages were seen in the submucosa of ulcerated appendices. In nine cases the granulomas were composed of epithelioid cells with Langhans' giant cells and central necrosis. Tuber-

Table Analysis of clinical and pathological findings in 180 appendices with unusual pathology

\begin{tabular}{|c|c|c|c|c|c|}
\hline \multirow[b]{2}{*}{ Diagnosis } & \multicolumn{5}{|c|}{ No of Cases } \\
\hline & $A$ & $B$ & $C$ & $D$ & Total \\
\hline $\begin{array}{l}\text { Granulomatous lesions: } \\
\text { Granuloma } \\
\text { Tuberculosis } \\
\text { Foreign body granuloma } \\
\text { Xanthogranuloma } \\
\text { Crohn's disease }\end{array}$ & 2 & 1 & $\begin{array}{l}9 \\
4 \\
2\end{array}$ & $\begin{array}{l}1 \\
6 \\
2\end{array}$ & $\begin{array}{r}10 \\
8 \\
6 \\
2 \\
1\end{array}$ \\
\hline $\begin{array}{l}\text { Parasitic diseases: } \\
\text { Enterobiasis } \\
\text { Schistomsomiasis } \\
\text { Trichuriasis } \\
\text { Ascariasis } \\
\text { Clonorchiasis }\end{array}$ & 1 & 2 & & $\begin{array}{r}25 \\
18 \\
6 \\
5 \\
1\end{array}$ & $\begin{array}{r}26 \\
20 \\
6 \\
5 \\
1\end{array}$ \\
\hline $\begin{array}{l}\text { Benign tumours and tumour li } \\
\text { Mucocele } \\
\text { Hyperplastic polyp } \\
\text { Adenoma } \\
\text { Mucinous cystadenoma } \\
\text { Carcinoid tumour } \\
\text { Leiomyoma } \\
\text { Neuroma }\end{array}$ & ns: & & $\begin{array}{l}6 \\
1 \\
3 \\
1 \\
9 \\
2\end{array}$ & $\begin{array}{l}2 \\
2 \\
1\end{array}$ & $\begin{array}{r}26 \\
1 \\
3 \\
6 \\
11 \\
2 \\
1\end{array}$ \\
\hline $\begin{array}{l}\text { Malignant lesions: } \\
\text { Adenocarcinoma } \\
\text { Secondary carcinoma } \\
\text { Malignant lymphoma } \\
\text { Lymphoblastic leukaemia }\end{array}$ & $\begin{array}{r}6 \\
24 \\
2 \\
1\end{array}$ & & & $\begin{array}{l}1 \\
1\end{array}$ & $\begin{array}{r}7 \\
25 \\
2 \\
1\end{array}$ \\
\hline $\begin{array}{l}\text { Miscellaneous lesions: } \\
\text { Endometriosis } \\
\text { Hirschprung disease }\end{array}$ & & $\begin{array}{l}6 \\
1\end{array}$ & & 3 & $\begin{array}{l}9 \\
1\end{array}$ \\
\hline Total & 48 & 10 & 37 & 85 & 180 \\
\hline
\end{tabular}

$\mathrm{A}=$ Gross abnormality pointing to pathological diagnosis described by surgeon or gynaecologist;

$\mathbf{B}=$ Diagnosis made on appendix was not contributory because of diagnosis made on prior or concurrent surgical specimens other than the appendix;

$\mathrm{C}=$ Non-consequential pathological diagnosis;

$\mathrm{D}=$ Important diagnosis made by routine pathological examination. 
culosis was presumptively diagnosed in eight cases and tuberculosis was suggested in the other case. While the clinical features and the gross pathological changes were highly characteristic of tuberculosis in two cases, acid fast bacilli could be identified in only one. Acid fast bacilli were identified in only one of the other seven cases. In the single case of Crohn's disease occurring in a 27 year old Chinese woman, the appendix showed no evidence of active disease. The appendix was examined along with the resected ileum and caecum. This case was included in our departmental file as a referred case from private doctors who had submitted a set of the slides from the hemicolectomy specimen for review two years after the surgery.

\section{PARASITIC DISEASES}

While in three of the 20 cases of schistosomiasis the disease was still active at the time of diagnosis, as judged by a florid granulomatous inflammation against the ova, the 17 other cases showed only calcific ova enclosed by hyaline fibrous tissue. In two patients the finding of ova in the appendix was noncontributory, as in one patient the diagnosis had been made on sigmoid colectomy one year before, and in the other, on concurrent right hemicolectomy specimen.

Of the five cases of ascariasis, a worm was grossly identified by the pathologist in only one case. Microscopic identification of ascaris were achieved in two cases, while ova were identified in the appendicular lumen in the remaining two cases. An adult Trichuris worm was identified macroscopically by a pathologist in one case. Five other cases of trichuriasis were diagnosed by finding sections of the parasite in the appendices. Of the 26 cases of enterobiasis, pinworms were grossly identified in only three appendices. The other cases were incidental findings in light microscopy. Clonorchis ova were identified in the lumen of one appendix.

\section{TUMOURS AND TUMOUR LIKE CONDITIONS}

Of the 26 cases of mucocele, the diagnosis was made on the mucin distended cystic dilatation of the appendices in nine cases. In two of these the appendices were ruptured and pseudomyxoma peritoni was present. In 11 other cases gross features suggestive of a mucocele were described by the pathologists before sections were studied microscopically. For the other six cases of mucocele the diagnosis was made only after examination by light microscopy. The single case of hyperplastic polyp and the three adenomas were microscopic findings in acutely inflamed appendices. Cystadenoma of the appendix with grossly identifiable mucin distended cavities was observed in three of the six cases, one of which was perforated, with pseudomyxoma peritoni.
The pathologists found mucinous cysts in two other cases. The remaining case was not noted to have gross features suggestive of the diagnosis. Nine cases of carcinoid tumours and the two cases of leiomyomas were microscopic findings. Small yellow intramural nodules were described by the pathologists and microscopy showed carcinoid tumours in two cases. A nodule occluding the appendiceal lumen was noted in the case of neuroma.

Spread to appendix in intra-abdominal and lymphoid malignancies was nearly always in the clinical context of disseminated malignancies, and the pathological findings in the appendix added little to the gross findings at operation. Primary ovarian carcinomas accounted for 12 cases. There were two bladder carcinomas, one oesophageal squamous cell carcinoma, one choriocarcinoma, and six colorectal carcinomas, two of which were of caecal origin with direct extension to the appendices. The primary tumours were unknown in three cases, including an unusual case of an acutely inflamed appendix of a 62 year old man which was found to have clumps of serosal adenocarcinoma cell deposits in the absence of any known primary or obvious tumour dissemination. The two other patients had diagnosed intraabdominal metastatic adenocarcinomas, the primaries of which were not mentioned in the biopsy request forms.

The appendicular spread in the two cases of malignant lymphoma was secondary to disseminated primary intestinal diseases. The patient with lymphoblastic leukaemia was a 21 year old man who had incidental acute appenditis during clinical leukaemic phase. The appendix and the enlarged mesenteric lymph nodes were infiltrated by leukaemic cells.

Among the seven patients with primary adenocarcinoma of the appendix, right hemicolectomy was performed in four of them for clinically malignant appendicular tumours. Widespread peritoneal tumour deposition was found in one of those patients who had undergone hemicolectomy. In one patient liver metastases were found at the time of laparotomy, and a polypoid growth in the appendix was identified by the surgeon: only the appendix was resected. In another patient, a 59 year old woman, the appendix was removed, ruptured mucocele was diagnosed, but the lesion turned out to be a mucoid carcinoma. The other patient was a 79 year old woman with an acutely inflamed and grossly dilated appendix. Light microscopy of the resected appendix showed the presence of a low grade adenocarcinoma infiltrating the muscle coat. It was not known whether right hemicolectomies were performed in these last two patients after primary malignancies in the appendix had been diagnosed by microscopy.

In six of nine patients with appendicular endo- 
metriosis the appendices were removed together with ovarian endometriotic cysts. In all cases the appendices were affected in the form of microscopic islands of serosal endometriosis and the diagnosis had little clinical impact.

The appendix in the case of Hirschprung disease was resected from a 3 year old boy whose disease was localised to the rectum, a fact established by a previous laparotomy. The appendix showed a normal population of ganglion cells.

The mean number of blocks taken in 300 consecutive cases of appendectomy in 1980 was $3 \cdot 2$ per case (median two blocks per case).

\section{Discussion}

Some centres only send resected appendices when the operative findings are inconclusive. ${ }^{2}$ The practice in this hospital has been to have all surgically resected appendices examined. Blocks are always taken for microscopy after gross examination by a pathologist. The mean number of blocks taken for each case was $3 \cdot 2$, based on a sample of 300 cases, and the median was two blocks per case. It is obvious that more lesions will be detected histologically if more sections are examined. Lesions of groups 1 and 2 consisted of 12333 entries of all the 12513 entries for appendicular lesions on file.

Examination of appendices over the 14 years showed a large variety of lesions, some of which would have been missed if the appendices had only been submitted when the operative findings were inconclusive. The diagnosis of some of these lesions was of great importance for further patient care. This is true for most of the parasitic diseases, some cases of granulomatous lesions, and benign or malignant tumours of the appendix.

Schistosomiasis in Hong Kong is caused by $S$ japonicum. Presumably all cases were imported from endemic regions in southern and central China, as Hong Kong does not provide a habitat for the paraasite to complete its life cycle. ${ }^{6}$ The disease is rare in Hong Kong. From the data obtained in this study, the disease occurred in $0 \cdot 16-0 \cdot 17 \%$ of the population. This is similar to the incidence of $0 \cdot 2 \%$ reported by Huang et al. ${ }^{7}$ In three of the 20 cases of schistosomiasis the presence of a florid granulomatous reaction against fresh ova showed that the disease was active. Chemotherapy aimed at eradicating living parasites would help prevent the release of more ova. Schistosomiasis might also be useful in explaining certain abnormal findings such as an enlarged spleen in these patients. In patients with inactive disease antihelminthics were probably not required as ova were no longer produced.

Ascariasis, enterobiasis, and trichuriasis can be effectively treated by chemotherapy and good personal hygiene. Microscopic examination of the appendix is an insensitive method of diagnosing clonorchiasis, which is the most prevalent parasitic disease in Hong Kong detected by stool examination. ${ }^{8}$ There is no satisfactory treatment for clonorchiasis. Acquisition of new parasites can be prevented if the patient refrains from eating raw or undercooked infected cyprinid fish. ${ }^{6}$

Xanthogranulomas consisting of collections of foamy macrophages were found in two appendices. The clinical importance of this change is not known. Neither of the pathologists pursued the case further by taking more blocks for section. In 12 other cases granulomatous inflammation was related to a variety of foreign materials, of which bewildering variety can be found in the appendices. ${ }^{1}$ It is unlikely that the diagnosis added anything to patient management in our 14 cases.

Enterobius granulomas in the appendical wall have occasionally been reported, ${ }^{9}$ and this diagnosis should be considered in the differential diagnosis of appendicular granulomas. While the practice of making a firm diagnosis of tuberculosis based on the finding of caseating epithelioid cell granulomas with or without being able to show acid fast bacilli can be criticised, the fact that this had been often done reflects the disease pattern in this community.

In Western societies the predominant role of tuberculosis with regard to Crohn's disease in the pathogenesis of appendicular granulomas is completely reversed. It is not known whether tuberculosis or other similar mycobacterial infections were actually responsible for the observed granulomatous lesions in the nine cases reported here. Crohn's disease is very rare in Hong Kong Chinese, and the case is one of the few reported. ${ }^{10}$

While it has been suggested that "mucocele of the appendix" should only be used in a clinical sense because the term has been used to cover several pathological entities, ${ }^{11}$ the pathologists of this department were still using it to denote any benign tumour like lesion of the appendix showing excessive accumulation of mucin. Since 1982 some pathologists in the department have made the distinction between hyperplastic polyp, adenomas, and mucinous cystadenoma of appendix. It is certainly useful to distinguish between hyperplastic polyp from other types of benign mucoceles as this lesion has very little potential to become malignant and has no known important clinical associations. Adenomas are premalignant ${ }^{12}$ and often associated with synchronous lesions in the large bowel. ${ }^{13}$

Carcinoid tumours were diagnosed in $0.088-0.096 \%$ of all appendices examined during the period studied. This incidence was much lower than 
the $0 \cdot 3-0 \cdot 7 \%$ reported by Moertel et al. ${ }^{14}$ As many appendicular carcinoids are microscopic lesions which are not grossly identifiable many cases could have been missed due to the limited number of sections examined in routine pathological examination. Carcinoid syndrome secondary to appendicular carcinoid tumour is extremely rare. ${ }^{15}$ None of the 11 cases of carcinoid tumours in this series had carcinoid syndrome.

In the present study primary carcinoma of the appendix occurred much less often than mucoceles and other benign epithelial tumours of the appendix. It is worth noting that one case of appendicular mucoid carcinoma was clinically labelled a mucocele. It is mandatory that any appendix which shows a dilated lumen filled with mucin should be examined microscopically to exclude malignancy. Pseudomyxoma peritoni could be found in association with benign or malignant appendicular tumours. ${ }^{16}$ All three cases in this series were related to a benign appendicular lesion.

When the appendix was affected in secondary malignancies, the primary lesions were most likely to be intra-abdominal or pelvic in origin. The case in which there was secondary spread to the appendix from a squamous cell carcinoma of oesophagus was unusual. Oesophageal carcinoma has rarely been found to affect the hollow viscera of the abdomen, even at postmorten examination. ${ }^{1718}$ Secondary malignant tumours in appendix nearly always occurred in the context of disseminated malignancies with known primaries. There was only one case in which clumps of metastatic carcinoma cells were found without a clinical diagnosis of tumour being indicated in the biopsy request form.

It is worth noting that no case of actinomycosis has been diagnosed despite the very large number of appendices examined.

Among the appendices studied over a period of 14 years were $85(0 \cdot 74 \%)$ with clinically important pathological findings likely to be missed if the appendices were not submitted to routine histopathological examination. Twenty cases showed gross abnormal features pointing to the respective pathological diagnosis. The remaining 65 cases were microscopically diagnosed and included seven cases of tuberculosis, 34 cases of intestinal roundworm infections, one case of clonorchiasis, 18 cases of schistosomiasis, one case each of primary and secondary carcinomas and three cases of endometriosis.

Our results show that routine histopathological examination of the appendix yields important clinical information in addition to the operative findings and should be undertaken in all cases.

\section{References}

1 Collin DC. 71,000 human appendix specimens. A final report, summarizing forty years` study. Am J Proctol 1963;14:265-81.

2 Gilmore OJA. Brodribb AJM, Browett JP, et al. Appendicitis and mistaking conditions. A prospective study. Lancet 1975;ii:421-4.

3 Hong Kong Commissioner for census and statistics. Hong Kong annual digest of statistics. Hong Kong: Government Printer, 1984.

4 Burkitt DP. The aetiology of appendicitis. $B r \quad J$ Surg 1971:58:695-9.

5 Committee on nomenclature and classification of disease. Systematized nomenclature of pathology. Chicago: College of American Pathologists, 1965.

$6 \mathrm{Ko} \mathrm{RC}$. Are there parasitic disease problems in man in Hong Kong? In: Ko RC, ed. Current perspectives in parasitic diseases. Hong Kong: Department of Zoology, University of Hong Kong, 1984:29-51.

7 Huang CT, Wong MM, Ma SL, Sum T. Postmortem and laboratory examinations for human intestinal helminths in Hong Kong. Trop Med 1969;11:134-6.

8 Duchastel P. Prevalence of stool parasites in Hong Kong residents and Indo Chinese refugees applying for emigration to Canada: retrospective study over two year period (1979-81). In: Ko RD, ed. Current perspectives in parasitic diseases. Hong Kong: Department of Zoology of University of Hong Kong, 1984:53-4.

9 Vinuela A, Fernandez-Rojo F, Martinez-Merino A. Oxyuris granulomas of pelvic peritoneum and appendicular wall. Histopathology 1979;3:69-77.

10 Chan CW, Lam KC, Ho JCI, Lai CL. Crohn's syndrome in the Chinese. American Journal of Proctology, Gastroenterology and Colon and Rectal Surgery 1984;35:8-17.

11 Higa E, Rosai J, Pizzimbono CA, Wise L. Mucosal hyperplasia, mucinous cystadenoma and mucinous cystadenocarcinoma of the appendix. A reevaluation of appendiceal "mucocele". Cancer 1973:33:1525-41.

12 Morson BC, Dawson IM. Adenomas and the AdenomaCarcinoma sequence. In: Morson BC, Dawson IM, eds. Gastrointestinal pathology. 2nd ed. Oxford: Blackwell Scientific publications, 1979:615-47.

13 Wolff $M$, Ahmed N. Epithelial neoplasms of the vermiform appendix (exclusive of carcinoid). II. Cystadenomas, papillary adenomas, and adenomatous polyps of the appendix. Cancer 1976;37:2511-22.

14 Moertel CG, Dockerty MB, Judd ES. Carcinoid tumors of the vermiform appendix. Cancer 1968;21:270-8.

15 Markgraf WH, Dunn TM. Appendiceal carcinoid with carcinoid syndrome. Am J Surg 1964;107:730-2.

16 Little JM, Halliday JP, Clenn DC. Pseudomyxoma peritonii. Lancet 1969;ii:659-63.

17 Anderson LL, Lad TE. Autopsy findings in squamous cell carcinoma of the esophagus. Cancer 1982;50:1587-90.

18 Sons HU, Borchard F. Esophageal cancer, autopsy findings in 171 cases. Arch Pathol Lab Med 1984;108:983-8.

Requests for reprints to: Dr KW Chan, Department of Pathology, University of Hong Kong, Queen Mary Hospital, Hong Kong. 